\title{
Erratum to: Perfusion imaging of brain gliomas using arterial spin labeling: correlation with histopathological vascular density in MRI-guided biopsies
}

\author{
Di Ningning ${ }^{1}$ - Pang Haopeng ${ }^{1}$ - Dang Xuefei ${ }^{2}$. Cheng Wenna ${ }^{3} \cdot$ Ren Yan $^{1}$. \\ Wu Jingsong ${ }^{4} \cdot$ Yao Chengjun ${ }^{4} \cdot$ Yao Zhenwei $^{1} \cdot$ Feng Xiaoyuan $^{1}$
}

Published online: 24 February 2017

(C) Springer-Verlag Berlin Heidelberg 2017

\section{Erratum to: Neuroradiology}

DOI 10.1007/s00234-016-1756-0

We note that there is an error in table 2 of the article "Perfusion imaging of brain gliomas using arterial spin labeling: Correlation with vascular density in MRI-guided biopsies", DOI: $10.1007 / \mathrm{s} 00234-016-1756-0$ :

Table 2

$\rho=0.267$ should read $\rho=0.567$

The online version of the original article can be found at http:// dx.doi.org/10.1007/s00234-016-1756-0.

\section{Feng Xiaoyuan}

xyfeng@shmu.edu.cn

1 Department of Radiology, Huashan Hospital Fudan University, Mid Urumqi Road, Shanghai 200040, People's Republic of China

2 Shang Hai Gamma Knife Hospital, Shanghai, People's Republic of China

3 Department of Pharmacy, Binzhou Medical University Affiliated Hospital, Binzhou, People's Republic of China

4 Department of Neurosurgery, Huashan Hospital Fudan University, Shanghai, People's Republic of China 